\title{
Reliability Methods for Shield Design Process
}

\author{
R.K. Tripathi and J.W. Wilson \\ NASA Langley Research Center, Hampton, VA 23681 USA \\ 757864 1467; r.k.tripathi@larc.nasa.gov
}

\begin{abstract}
Providing protection against the hazards of space radiation is a major challenge to the exploration and development of space. The great cost of added radiation shielding is a potential limiting factor in deep space operations. In this enabling technology, we have developed methods for optimized shield design over multi-segmented missions involving multiple work and living areas in the transport and duty phase of space missions. The total shield mass over all pieces of equipment and habitats is optimized subject to career dose and dose rate constraints. An important component of this technology is the estimation of two most commonly identified uncertainties in radiation shield design, the shielding properties of materials used and the understanding of the biological response of the astronaut to the radiation leaking through the materials into the living space. The largest uncertainty, of course, is in the biological response to especially high charge and energy (HZE) ions of the galactic cosmic rays. These uncertainties are blended with the optimization design procedure to formulate reliability-based methods for shield design processes. The details of the methods will be discussed.
\end{abstract}

\section{INTRODUCTION}

Following the Apollo mission, Human Exploration and Development of Space (HEDS) activity has been limited to low Earth orbit (LEO). With the advancing International Space Station (ISS) providing a first level of infrastructure for human exploration, preparation for additional HEDS activity beyond LEO is the natural extension of the current space program. Without dispute, providing protection for astronauts and equipment from the hazards of space radiation is a critical enabling technology (for HEDS) and one of NASA's two highest priorities (O'Keefe, 2002). Faced with a limited budget and an expanding space exploration program, the old way of doing business is inadequate and NASA requires revolutionary technologies to make advances.

Radiation health-risk mitigation in the advancing HEDS program will utilize an array of methods to control adverse health effects. These are well defined by Cucinotta et al. (2001) and includes advanced propulsion systems, crew selection, biological countermeasures, and operational approaches. Fundamental to risk mitigation is the advance in knowledge of the radiation risk coefficients for which large design margins are presently required. As suggested by Cucinotta et al. (2001), reducing the risk uncertainty is a "cost effective approach using data collection and research" to possibly add years to the careers of space workers. It is the purpose of the present development to incorporate our current state of knowledge of radiation risks into the shield design process. Such a development will allow evaluation of radiation risk-uncertainty impact on shield design, allow the design of reliable shielding for future missions, and quantify the advantage of future research in terms of strict engineering practice.

In view of the uncertainties in biological response to cosmic radiation and lack of a definitive criterion for acceptable levels of risks for deep space missions reliability based methods for radiation shielding and protection, as discussed herein, are of enormous importance to human exploration and development of space. Even if a definitive criterion were available for deep space missions, uncertainties in biological response to deep space radiations would remain with us for a long time. Hence, the reliability based methods for the design process for radiation protection and shielding will continue to play a significant role for space exploration for many years to come.

The NCRP (1989) suggestion that the LEO exposure limits be used as a guideline for deep space mission studies, uncertainties in those limits as applied to deep space would be a major driver in mission costs (Wilson et al., 1997). 
Although the basic physics of shield evaluation has made great progress (Cucinotta et al. 1998, Shinn et al 1998, Wilson et al. 1998, Golovchenko et al. 2002), the primary uncertainty in shielding estimates result from the large uncertainty in biological response to high charge and energy (HZE) ions of the galactic cosmic rays (Cucinotta et al. 2001). In the present study, we will begin the development of a software framework to evaluate the best solution to shielding problems under the limitations of biological uncertainty. In essence, the distribution of probable biological response (related to uncertainty) will be used to evaluate the phase-space of probable shielding solutions. The best shield solution corresponding to a specified confidence level of biological risks will provide a means of entering this uncertainty into the design process. Thusly, design reliability is assured within current knowledge by robust design processes that can be specified to a given level of reliability. To make such methods practical, each probable design needs to be optimized with respect to onboard materials and across the mission architecture (Wilson et al. 2001, 2002, Tripathi et al. 2001, 2002). Optimization frameworks are developing and preliminary analysis of reliability based methods is the focus of the present paper.

The development of reliability based methods are dependent on shield analysis software and the ability to calculate the quantities required for reliability design processes and further enable design optimization across mission architectures. An enabling technology to accomplish such tasks is the development of highly efficient deterministic computational procedures (Wilson et al. 1991, Tripathi et al. 2001, Wilson et al. 2002) for shield evaluation able to provide high-quality estimates of the improbable events so important to biological injury (Wilson et al. 1995). At a practical level, this means evaluation of radiation field quantities within complex structures within minutes to enable optimization procedures (Qualls et al. 2001, Nealy 2003).

In the present paper, we will first review the underlying quantities to be considered and their implementation into the design process. We will then use this development to examine a typical Mars mission and evaluate the impact of reliability design methods compared to nominal methods suggested by the NCRP (1989). In this application we will only consider the travel to and return from Mars since a short stay on the surface with the added protection of the atmosphere minimizes the shielding requirements on the surface. Clearly future developments will require a more complex mission scenario and optimization across a more complex array of habitats and vehicles. We will then review the implications of the reliability design process on shield design software and computational requirements on quantities to be evaluated to enable reliability-based design within a multidisciplinary design environment.

\section{SPACE RADIATION ENVIRONMENT AND BIOLOGY}

The basic requirement on astronaut exposure limitations established by the National Council on Radiation Protection is that radiation induced excess fatal cancer risks from career exposures are to be limited to be less than 3 percent and early radiation syndrome (nausea, vomiting...) is to be avoided (NCRP 1989, 2001). Qualitatively, this is similar to the requirements for terrestrial radiaiton workers. The radiation environment in low Earth orbit (LEO) is of such a character that career radiation exposure limits have been given by the NCRP in terms of a local tissue related quantity known as dose equivalent (Seivert, NCRP 2001) given by

$$
H=\int Q(L) D_{L} d L
$$

where $\mathrm{Q}(\mathrm{L})$ is the quality factor (ICRP 1991) relating to the difference in induced risk of differing particle types delivering the same dose and $\mathrm{D}_{\mathrm{L}}$ is the dose (Gray) from components with linear energy transfer between $\mathrm{L}$ and $\mathrm{L}+$ dL. Note that equation (1) breaks the convention of the ICRP (1991) who have recommended radiation field weighting factors for estimation of fatal cancer risks which does not depend on the local tissue field. The argument given by the ICRP is that the uncertainty introduced through such a nonlocal approximation is indicative of the uncertainty in risk estimation methods in distinction to equation (1) that gives the appearance of a quantified risk. The approach by the NCRP in recommending equation (1) allows a quantitative treatment of uncertainty as noted by Cucinotta et al. (2001) and enables the development of reliability based methods, as we will show herein. The LEO environment consists mainly of particles of a restricted range of LET for which data on relative biological effectiveness was reasonably known (see Fig. 1) and dose limitations are given by the NCRP in this case (2001). In the figure, the eye exposure is given as an example and it is seen that the dominant component is the trapped protons. At lower altitudes the trapped protons decline leaving the cosmic ray exposures somewhat more important. 
The RBE associated with early radiation syndrome have been recently defined by the NCRP (2001) to relate to a new quantity Gy-Eq in terms of field quantities (nonlocal quantity) as

$$
\text { Gy-Eq }=\sum_{\mathrm{i}} \mathrm{RBE}_{\mathrm{i}} \mathrm{D}_{\mathrm{i}}
$$

where $R B E_{i}$ is the relative biological effectiveness of the ith field component resulting in dose $D_{i}$ to the specific tissue. Limitations on dose equivalent and Gray equivalent have been given by the NCRP for LEO operations as given in tables 1 and 2.

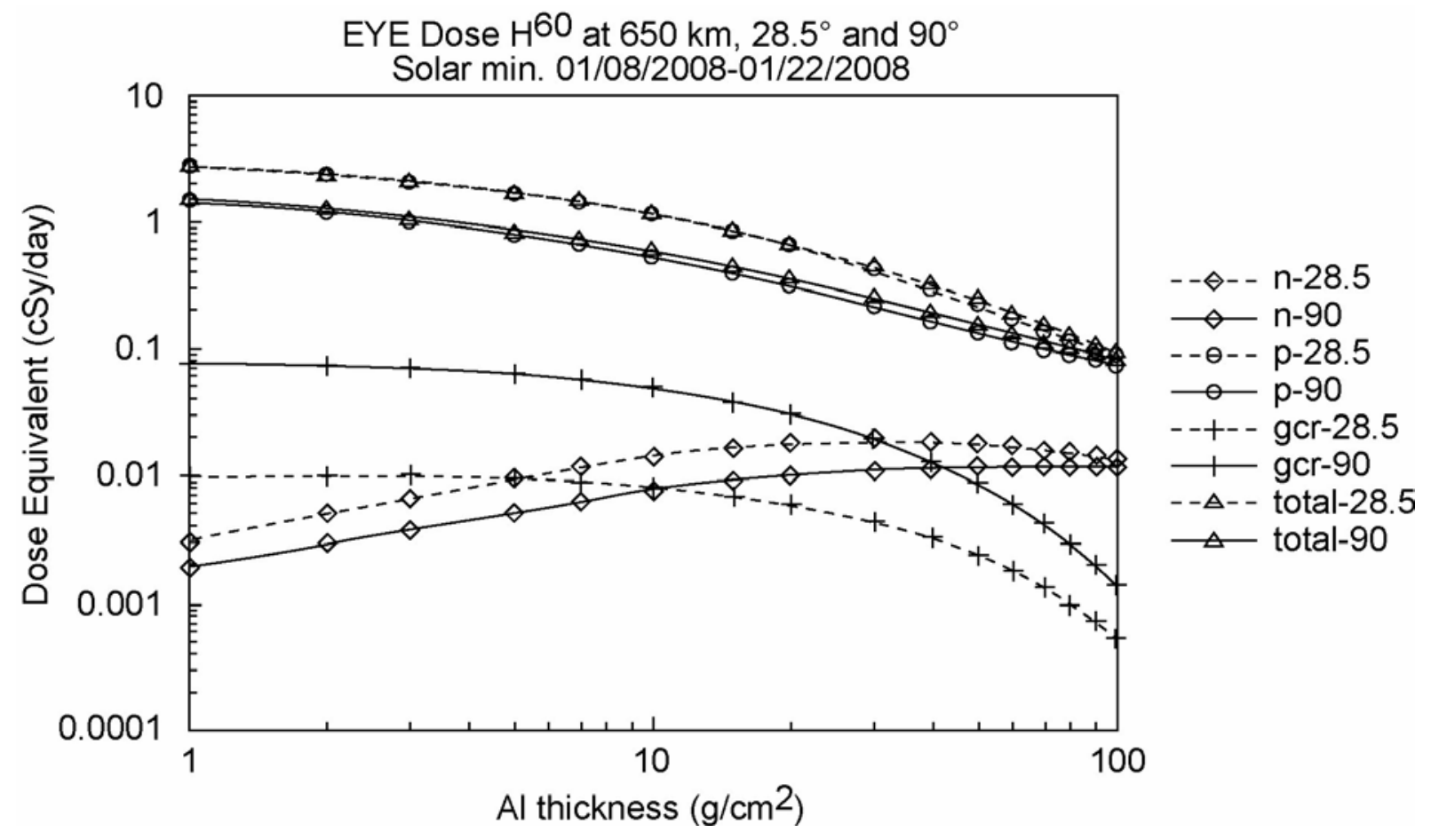

FIGURE 1. Eye Dose Equivalent at $650 \mathrm{~km}$ with Aluminum Shielding.

The present exposure constraints used in the space program are recommended for low Earth orbit (LEO) operations by the National Council on Radiation Protection (NCRP 2001) and approved by the NASA Administrator and OSHA. There are no limits for deep space operations due to the unusual composition of the GCR and the resultant uncertainties in associated health risks (Cucinotta et al. 2001). The LEO limits are given for the three critical organs of skin, ocular lens, and blood forming organ (BFO) in table 1 and table 2 applies to whole body exposure and implies appropriate average over the body tissues (NCRP 2001).

TABLE 1. Recommended Organ Dose Equivalent Limits for All Ages.

\begin{tabular}{|c|c|c|c|}
\hline & BFO, Sv & Eye, Sv & Skin, Sv \\
\hline Career & See Table 2.2 & 4.0 & 6.0 \\
\hline Annual & 0.50 & 2.0 & 3.0 \\
\hline 30 Days & 0.25 & 1.0 & 1.5 \\
\hline
\end{tabular}

TABLE 2. Career Whole Body-Dose Equivalent Limit (Sv) for Lifetime Excess Risk of Fatal Cancer of Three Percent as a Function of Age at Exposure.

\begin{tabular}{|l|l|l|l|l|}
\hline Age & 25 & 35 & 45 & 55 \\
\hline Male & 0.7 & 1.0 & 1.5 & 2.9 \\
\hline Female & 0.4 & 0.6 & 0.9 & 1.6 \\
\hline
\end{tabular}


In addition to the trapped radiations and the galactic cosmic rays able to penetrate the geomagnetic field to LEO, there are occasional solar particle events able to penetrate the geomagnetic field. The solar particle source is mainly composed of protons of similar quality as the trapped protons and the limitations in tables 1 and 2 are applicable. The implications of the galactic cosmic ray exposures on LEO operations have not been fully evaluated with respect to exposure limitations.
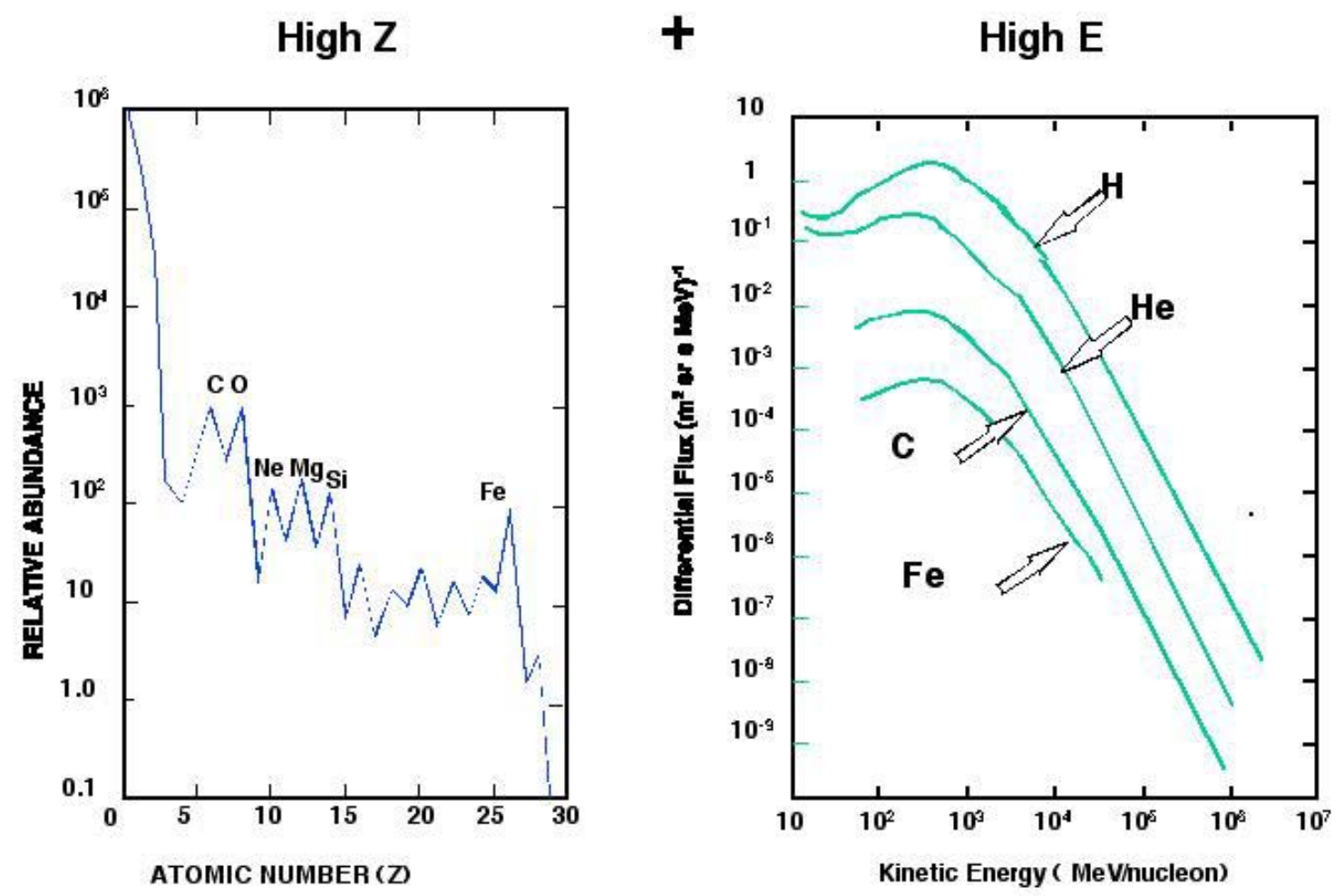

"HZE"

FIGURE 2. Galactic Cosmic Ray Spectra in Charge (Z) and Energy (E).

Beyond the geomagnetospheric, the galactic cosmic rays (GCR) are the dominant long-term exposure hazard. Generally for deep space operations, the provision of protection from galactic cosmic rays will provide most, if not all, the protection required from solar particle events. The GCR are ions of every known element with spectra spanning rather modest energies to very high energies (Fig. 2). Although mostly protons and helium ions, the highenergy heavy ions or HZE ions deliver a large fraction of the dose equivalent. The LET of a given ion is proportional to the square of the charge and a very broad range of LET is encountered beyond LEO for which there is little biological data.

As given in equation (1), the cancer risk is related to the LET distribution of the dose modified by the quality factor $\mathrm{Q}(\mathrm{L})$. Control of cancer risk is accordingly to control the LET distribution of particles and in this not all materials are equal (Wilson et al. 1995). The LET is dependent on ion type and energy that is modified by fragmenting the ions in nuclear processes to different ion types and slowed in speed by atomic interactions. Both processes depend on the composition of the material. A third related process is the generation of new particles by collision with the complex nuclei in the shield material. One must balance these three processes in selection of material with which to construct the shielding to protect the astronauts (Wilson et al. 1995, 2002). 
The most efficient shielding material is provided by liquid hydrogen (Wilson et al. 1995). It shows the greatest attenuation of high LET components with minimal addition of troublesome secondary radiations (see Fig. 3). Also relatively efficient is water also shown in Fig. 3 for comparison. Aluminum has been shown to be a poor material

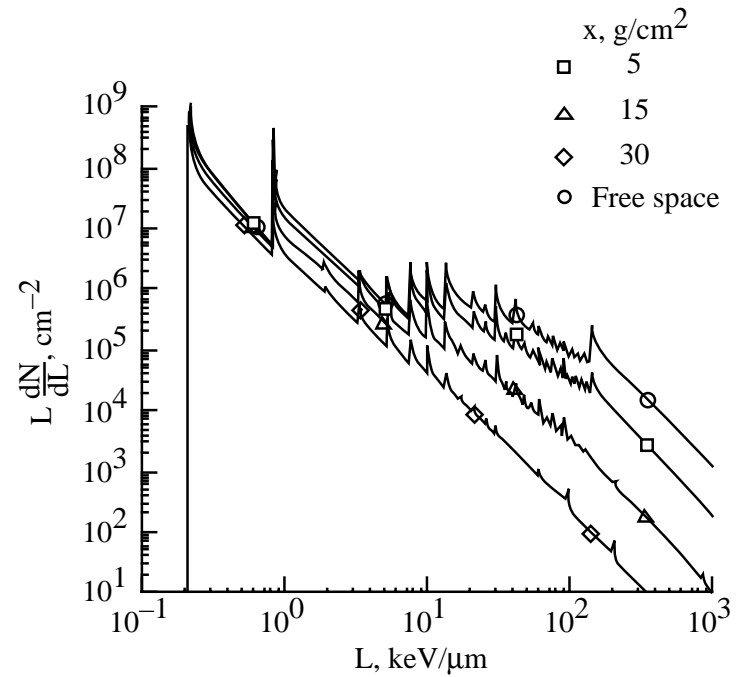

(a) Liquid $\mathrm{H}_{2}$

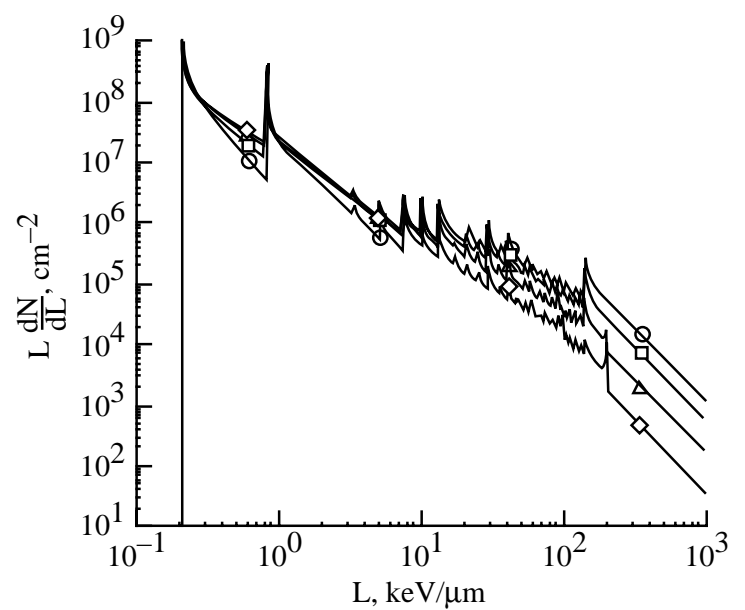

(b) Water

FIGURE 3. LET Spectral Dependence on Shielding Material: (a) Liquid Hydrogen and (b) Water.

for spacecraft construction since secondary radiations create an additional hazard and any improved protection occurs only at very large depths (Wilson et al. 1995). Materials with even higher atomic numbers than aluminum ( $Z$ $=13$ ) are progressively more hazardous. Even local in situ materials need to be used with care because of the nature of the secondary radiations produced. The augmentation of local materials with polymer binders can help in controlling secondary radiations (Kim et al. 2001) but requires more development, testing, and complete evaluation for the specific application. Some of the required tools for this evaluation are the subject of the present paper.

In application of equation (1) in setting exposure limits, the NCRP assumed a linear relation of excess fatal cancer risk to dose equivalent as is appropriate for low dose and low dose rate exposures given by

$$
\mathrm{R}(\mathrm{A}, \mathrm{G})=\left[\mathrm{r}_{0}(\mathrm{~A}, \mathrm{G}) / \mathrm{DDREF}\right] \int \mathrm{D}_{\mathrm{L}} \mathrm{Q}(\mathrm{L}) \mathrm{dL}
$$

where $r_{0}(A, G)$ is the age $A$ and gender $G$ dependent risk coefficient for the high dose rate exposures in the a-bomb detonations of WW-II. The quality factor Q(L) is defined by the ICRP (1991) according to observed RBE factors.

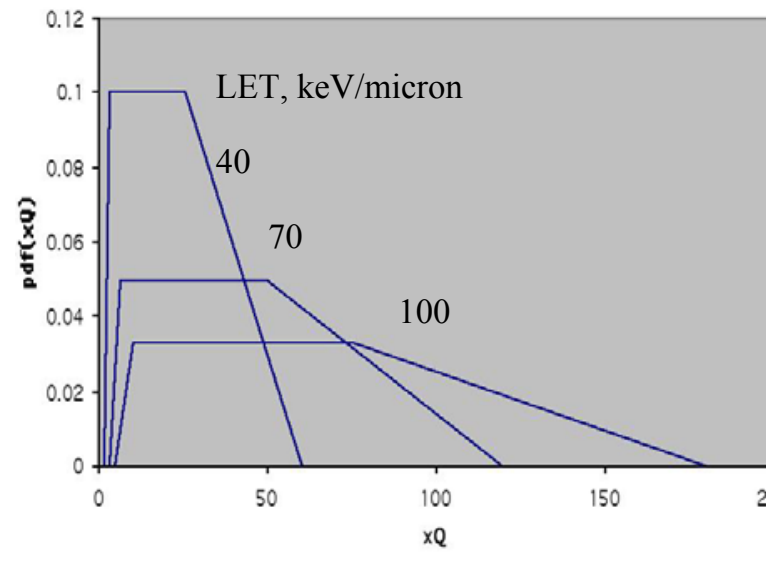

FIGURE 4. Probability Distribution of Quality Factor $X_{Q}$.

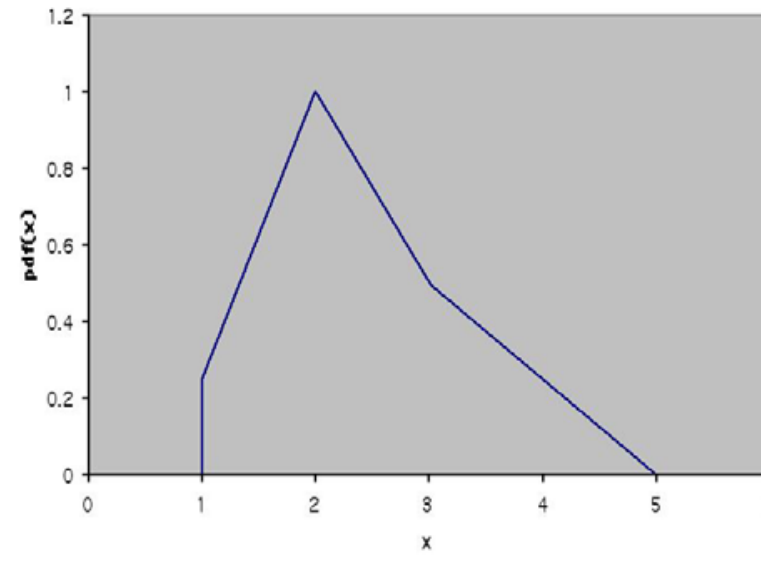

FIGURE 5. Probability Distribution of $\mathrm{X}_{\mathrm{DDREF}}$. 
The dose and dose rate effectiveness factor, DDREF, was derived from animal experiments and taken by the NCRP as a factor of two in the setting of dose limits (NCRP 1997). The uncertainty in application of equation (3) is discussed by Cucinotta et al. (2001). In the present study we will consider the two main sources of uncertainty being that in $\mathrm{Q}(\mathrm{L})$ and $\mathrm{DDREF}$.

To illustrate the approach, we follow Cucinotta et al. (2001) in modeling the uncertainty in equation (3) whereby the nominal values of $\mathrm{Q}(\mathrm{L})$ and DDREF are replaced by stochastic variables $\mathrm{X}_{\mathrm{Q}}$ and $\mathrm{X}_{\mathrm{DDREF}}$ as

$$
\mathrm{R}(\mathrm{A}, \mathrm{G}, \mathrm{\kappa})=\left[\mathrm{r}_{0}(\mathrm{~A}, \mathrm{G}) / \mathrm{X}_{\mathrm{DDREF}}\right] \int \mathrm{D}_{\mathrm{L}} \mathrm{X}_{\mathrm{Q}} \mathrm{dL}
$$

where the $\kappa$ is a statistical variable denoting $R(A, G, \kappa)$ to be a member of a population of possible biological response models associated with the uncertainty in $Q(L)$ and DDREF. The probability function of $X_{Q}$ depends on the LET as shown in Fig. 4 as found by Cucinotta et al. (2001) after examining a broad range of RBE measured over a broad range of LET. Similarly, the probability distribution function of $X_{\text {DDREF }}$ is given by the NCRP (1997) as shown in Fig. 5.

We will now examine the impact of these uncertainties on the design of spacecraft in simple geometry to begin to understand the impact of biological uncertainty on the mission design. The first step in the process has already been taken by Cucinotta et al. (2001). An example is that shown in Fig. 6 where the range of risk within an aluminum shell shield is shown as a function of shield thickness. In the figure is shown the range of risks implied by uncertainty in the risk model factors including the uncertainty in the physical parameters associated with shield evaluation. Again, in the present study we consider only the two uncertainty factors in Figs. 4 and 5.

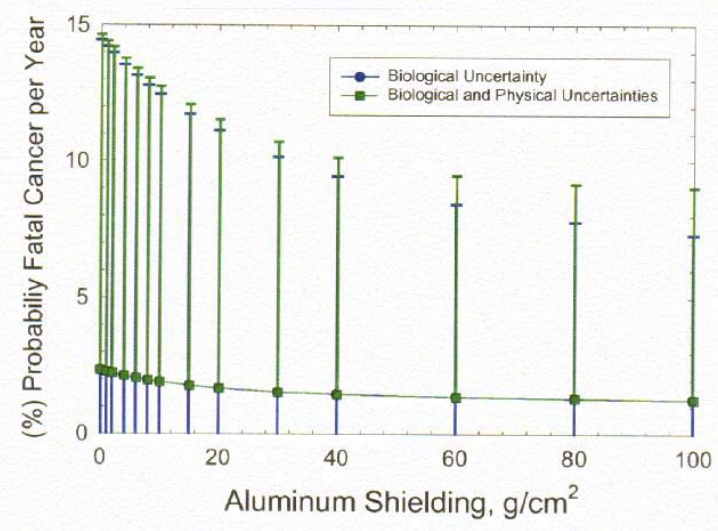

FIGURE 6. Range of Risk Uncertainty within an Aluminum Shell (Cucinotta et al. 2001).

\section{RELIABILITY SHIELD DESIGN}

It is desirable to setup the reliability design problem so as to most easily integrate into the mission architecture software derived on the basis of nominal risk coefficients. In that formalism, we used the nominal limits given by the NCRP (2001) based on nominal risk coefficient used in LEO constraints found in tables 1 and 2. We rewrite equation (4) as

$$
\mathrm{R}(\mathrm{A}, \mathrm{G}, \kappa)=\left[\mathrm{r}_{0}(\mathrm{~A}, \mathrm{G}) / \mathrm{DDREF}\right] \int \mathrm{M}(\mathrm{L}, \kappa) \mathrm{D}_{\mathrm{L}}(\mathrm{x}) \mathrm{dL}
$$

where DDREF is two as concluded by the NCRP (1997) and $\mathrm{D}(\mathrm{L}, \mathrm{x})$ is the distribution of dose over LET within a spherical shell of thickness $x$ assuming the physics related uncertainties are negligible. The function $M(L, \kappa)$ is an off-nominal LET dependent modifying function in which all biological uncertainty resides and is numerically equal to $\mathrm{Q}(\mathrm{L})$ for the nominal risk. In this way the uncertainty is assigned to the evaluation of "dose equivalent" but is 
otherwise directly related to the dose limits in table 2 . The dose equivalent in this context is given as a stochastic variable by

$$
H(x, \kappa)=\int M(L, \kappa) D_{L}(x) d L
$$

Then the requirement of limiting the lifetime excess fatal cancer risk given by equation (5) to less than three percent is equivalent to limiting the value of dose equivalent as given by equation (6) to be less than the nominal exposure limits in table 2 .

In its simplest form the design process can be implemented as follows. For a given shield thickness $\mathrm{x}$, there is a stochastic sequence of associated risks $R(A, G, \kappa)$. The shielding is acceptable to a given confidence level (CL) when $\mathrm{CL}$ percent of the risk sequence lies below three percent. One must seek to find a value of $\mathrm{x}$ that satisfies this requirement. If a mission using this shield configuration was sent on such a mission then we are confident that the excess fatal cancer risk to the astronaut would not exceed three percent as a result of the mission with a level of confidence CL. The present formulation is the simplest form of the method and is next to be combined with the mission architecture profile optimization and multidisciplinary optimization procedures.

\section{AN EXAMPLE APPLICATION}

To demonstrate the method, we consider a two-year mission to Mars with a mixed crew of six. The optimum volume of living space is taken as $114 \mathrm{~m}^{3}$ and crew age set at the youngest female. It is assumed that the living

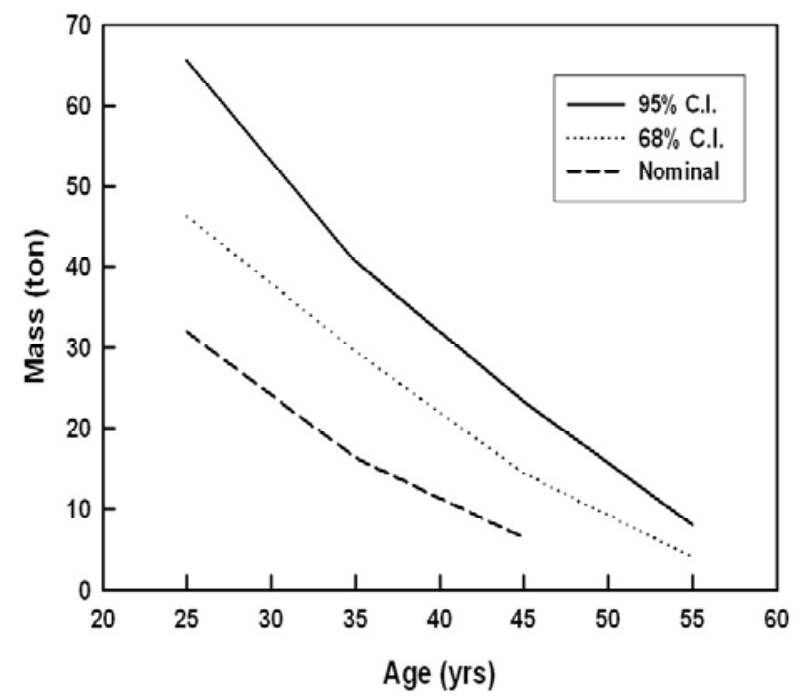

(a) Liquid $\mathrm{H}_{2}$

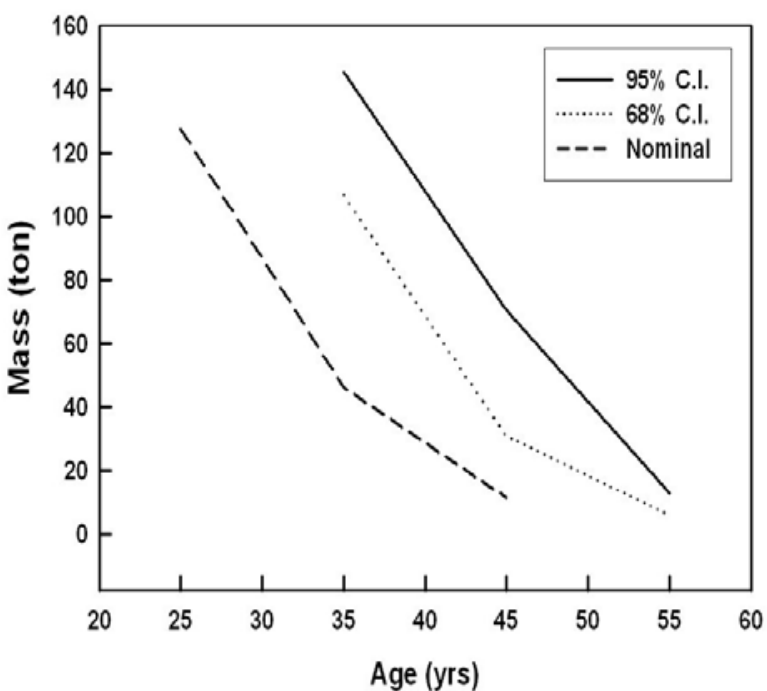

(b) HGNF

FIGURE 7. The Stochastic Design Space for a Two Year Mars Mission for Two Highly Efficient Shield Materials.

space is a right circular cylinder $2.2 \mathrm{~m}$ high. Assuming the walls to be a pressure vessel containing either liquid hydrogen (LH2) or hydrogenated graphite nanofibers with herringbone structure (HGNF) the design mass as a function of age is shown in figure 7. Although this is not the exact geometry and only the shield wall is represented we see a large impact of uncertainty on the design.

In our prior studies of optimization using nominal risk coefficients, only the LH2 and HGNF showed promise as a shield material for such long duration missions in deep space. Recalling that HGNF is a factor of 4 to 6 more efficient at protecting the astronaut than aluminum (Wilson et al. 2001) it is clear that few material options are available for future space mission and other methods such as biological countermeasures will play a pivotal role. At 
the very minimum from an engineering design point of view, reliability based methods must be implemented to accurately portray the shielding component of risk mitigation in mission design.

\section{DISCUSSION}

Protecting the astronaut from the adverse effects of space radiation will require a triage of which shielding is only one aspect. Since the radiation within the cabin space is the initiating insult the cabin shield effectiveness is the first line of mitigation of adverse health effects. Because of the great uncertainty in biological response will be with us into the foreseeable future, reliability based design methods will play a pivotal role in mission analysis and design. As such there are several requirements that supporting computational software will have to meet.

The computational procedures will have to provide the distribution of dose in specific tissues as a function of LET in order to have a sensible implementation of reliability based methods. The evaluation of the tissue specific dose distribution will have to be implemented so as to allow rapid evaluation of design options to enable optimization processes. If stochastic computational procedures are used then great care must be given to assuring that the biological important but rare events are represented to high statistical accuracy and that statistical noise in solution be eliminated to assure compatibility with gradient driven optimization procedures. Statistical noise cannot be allowed to drive autonomous optimization procedures to false solutions.

\section{CONCLUSIONS}

We have examined an approach to introduce reliability based design methods into shield evaluation as a means to assess and control the uncertainties in shield design. Application to a Mars mission displays a large impact on the design outcome. Computational procedures based on deterministic solution of the Boltzmann equation are well suited for such procedures allowing optimization processes to be implemented, evaluation of biologically important rare events, and rapid analysis of possible shield optimization outcomes resulting from the biological model uncertainty parameter space. Shield design studies based on nominal biological response models are highly questionable in their result and may lead to designs in which astronaut risk are much higher than anticipated on the basis of such models. Reliability based methods result in designs for which astronaut risk within the limitations of current knowledge is well controlled in the design process.

\section{REFERENCES}

Cucinotta, F.A., Wilson, J.W., Shinn J.L., Tripathi, R.K., “Assessment and Requirements of Nuclear Reaction Databases for GCR Transport in the Atmosphere and Structures," Adv. Space Res., Vol. 21, No. 12, pp. 1753-1762 (1998).

Cucinotta, F.A., W. Schimmerling, J.W., Wilson, L.E. Peterson, G.D. Badhwar, P. Saganti, J.F. Dicello, 'Space radiation cancer risks and uncertainties for Mars missions,', Johnson Space Center, JSC- 2925 (2001).

Golovchenko, A.N., Skvarc, J., Yasuda, N., Giacomelli, M., Tretyakova, S.P., Illic, R., Bimbot, R., Toulemonde, M., Murakami, T., "Total charge-changing and partial cross-section measurements in the reactions of $\approx 110-250 \mathrm{MeV} /$ nucleon $12 \mathrm{C}$ in carbon, paraffin, and water.' Phys. Rev., C 66, 014609 (2002).

ICRP, The 1990 Recommendations of the International Commission for Radiological Protection, ICRP Report 60, Pergamon Press, Oxford, UK, (1991).

Kim, M.-H. Y., Thibeault, S.A., Kiefer, R.L., Wilson, J.W., Singleterry, R.C., Moore, J., Huff, H., and Wilkins, R.,'Fabrication and Testing of Habitat Components Using In Situ Materials for Martian Exploration,' $46^{\text {th }}$ International SAMPE Symposium and Exhibition, Long Beach, California, May 6-10, 2001.

NCRP, Uncertainties in Fatal Cancer Risk Estimates Used in Radiation Protection, NCRP Report No. 126 (1997).

NCRP, Guidance on Space Exposures in Space, National Council on Radiation Protection, Bethesda, NCRP Report No. 98 (1989).

NCRP, Radiation Protection Guidance for Activities in Low-Earth Orbit, National Council on Radiation Protection, Bethesda, NCRP Report No. 132, (2001).

Nealy, J.E., Anderson, B.A., Wilson, J.W., and Qualls, G.D.,"High-Speed Computational Applications for Space Radiation Shielding Analysis," in the proceedings of Space Technology and Applications International Forum (STAIF 2003), edited by M.E-Genk, AIP Conference Proceedings 608, Melville, New York, 2003. 
O'Keefe, S., '’Admistrator O'Keefe pitches his vision for NASA,' http://www.spaceflightnow.com/news/n0203/27okeefe/.

Qualls, G.D., Wilson, J.W., Sandridge, C.A., Cucinotta, F.A., Nealy, J.E., Heinbockel, J.H., Hugger, C.P., VerHage, J., Anderson, B.M., Atwell, W., Zapp, N., and Barber, R.,' International Space Station Shielding Model Development," SAE 2001-01-2370 (2001).

Shinn, J.L., Cucinotta, F.A., Simonsen, L.C., Wilson, J.W., Badavi, F.F., Badhwar, G.D., Miller, J., Zeitlin, C., Heilbronn, L., Tripathi, R.K., Clowdsley, M.S., Heinbockel, J.H., Xapsos, M.A., "Validation of a Comprehensive Space Radiation Transport Code," IEEE Transactions on Nuclear Science, Vol. 45, No. 6, Part 1, pp. 2711-2719 (1998).

Tripathi, R.K., Wilson, J.W., Cucinotta, F.A., Nealy, J.E., Clowdsley, M.S., Kim, M.-H. Y., "Deep space mission shielding optimization," SAE 2001-01-2326 (2001).

Tripathi, R.K., Simonsen, L.C., Nealy, J.E., Troutman, P.A., Wilson, J.W., "Shield optimization in simple geometry for the Gateway concept," SAE 2002-01-2332 (2002).

Wilson, J. W., Townsend, L.W., Schimmerling, W., Khandelwal, G.S., Khan, F., Nealy, J.E., Cucinotta, F.A., Simonsen, L.C., Shinn, J.L., and Norbury, J.W., Transport Methods and Interactions for Space Radiations. NASA RP-1257 (1991).

Wilson, J.W., Kim, M.; Badavi, F. F., Thibeault, S. A., Cucinotta, F. A., Shinn, J. L., and Kieffer, R.,' Issues in Protection From Galactic Cosmic Rays," Health Phys. 68, 50-58 (1995).

Wilson, J.W., Miller, J., Konradi, A., Cucinotta, F.A., "Shielding Strategies for Human Space Exploration," NASA CP-3360 (1997).

Wilson, J. W., Cucinotta, F. A., Tai, H., Shinn, J. L., Chun, S. Y., Tripathi, R. K., and Shiver, L., "Transport of Light Ions in Matter," Adv. Space Res. 21(12), 1763-1771 (1998).

Wilson, J.W., Shinn, J.L., Tripathi, R.K., Singleterry, R.C., Clowdsley, M.S., Thibeault, S.A., Cheatwood, F.M., Schimmerling, W., Cucinotta, F.A., Badhwar, G.D., Norr, A.K., Kim, M.Y., Badavi, F.F., Heinbockel, J.H., Miller, J., Zeitlin, C., Heilbronn, "Issues in deep space radiation protection," Acta Astronautica 49, 289-312 (2001).

Wilson, J.W., Korte, J.J., Heinbockel, J.H., Clowdsley, M.S., Badavi, F.F., Troung, A., "Ionizing Radiation: Multifunctionality and MDO Processes," SAE 2002-01-2334 (2002). 